\title{
A Demonstration of Caregiver-Implemented Functional Analysis of Inappropriate Mealtime Behavior via Telehealth
}

\author{
Ashley S. Andersen ${ }^{1}$ (D) $\cdot$ Bethany A. Hansen ${ }^{1}$ (D) $\cdot$ Kristin L. Hathaway ${ }^{1} \cdot$ Lindsey A. Elson $^{1}$ \\ Accepted: 26 May 2021 / Published online: 8 July 2021 \\ (C) This is a U.S. government work and not under copyright protection in the U.S.; foreign copyright protection may apply 2021, corrected publication 2021
}

\begin{abstract}
Treatment for pediatric feeding disorders is imperative, so clinicians should alter the assessment and treatment process, making it is possible for caregivers to deliver via telehealth. Clinicians must first demonstrate that caregivers can conduct initial assessments in this format. We extended the literature on functional analysis of inappropriate mealtime behavior by demonstrating that caregivers can implement functional analysis procedures via telehealth to identify maintaining variables of inappropriate mealtime behavior. Clinicians determined that escape, attention, and tangibles and escape maintained inappropriate mealtime behavior during solids and liquids meals, respectively.
\end{abstract}

Keywords functional analysis $\cdot$ inappropriate mealtime behavior $\cdot$ pediatric feeding disorder $\cdot$ telehealth

The COVID-19 pandemic introduced challenges for behavior analysts who provide essential services, requiring them to weigh the benefits against the risks (Cox et al., 2020). Some clinics had discontinued face-to-face services or, due to medical histories placing a child at high risk, children were unable to receive services in a clinical setting. Without proper protective equipment, in-person feeding services may not align with the Centers for Disease Control's (CDC, 2020) recommendations to social distance and wear face coverings, because the feeder must be close to the child to feed bites or drinks and the child must have their mouth exposed to accept food or liquid into their mouth. When appropriate, providing services via

- Clinicians with experience assessing and treating feeding problems should consider important variables to determine the appropriateness of telehealth services.

- With ongoing coaching, caregivers implemented functional analysis procedures with high procedural integrity.

- Clinicians identified maintaining variables of inappropriate mealtime behavior from the caregiver-fed functional analyses via telehealth.

- When providing services for children with feeding problems via telehealth, clinicians should ensure involvement from an interdisciplinary team, so all variables contributing to the feeding problems are addressed.

Bethany A. Hansen

bethany.hansen@unmc.edu

1 University of Nebraska Medical Center, Munroe-Meyer Institute, 444 South 44th Street, Omaha, NE 68105, USA telehealth gives clinicians and families an option that aligns with the CDC's recommendations (Cox et al., 2020).

Children with severe feeding problems, such as gastrostomy-tube (G-tube) dependence, are often referred for intensive intervention. These children are at risk for inadequate caloric intake, dehydration, and nutritional deficiencies (Volkert \& Piazza, 2012). Intensive programs allow children to receive a high dosage of therapy with access to an interdisciplinary team and the capability for behavior analysts to use systematic approaches. Identifying methods for continuing intensive intervention for feeding problems and providing access to telehealth services is essential.

Peterson et al. (2021) demonstrated that treatment effects established in a clinic setting could transfer to telehealth with minimal impact on the child's performance. Likewise, researchers have demonstrated that intervention for food selectivity (Bloomfield et al., 2019) or advanced feeding skills (e.g., chewing, Volkert et al., 2014) can be delivered via telehealth. However, initial assessment and treatment for food or liquid refusal via telehealth have not yet been demonstrated.

Conducting a functional analysis to identify environmental variables that maintain the child's inappropriate mealtime behavior (defined below) is imperative. Previous researchers evaluated functional analysis procedures for inappropriate mealtime behavior and demonstrated convergent validity with subsequent treatment evaluations (e.g., Bachmeyer et al., 2009). Some research has shown treatment must target all identified functions to produce clinically significant changes 
in inappropriate mealtime behavior and acceptance of bites or drinks, demonstrating the importance of a pretreatment functional analysis (Bachmeyer et al., 2019).

Inappropriate mealtime behavior may pose challenges for caregivers to conduct a functional analysis in a telehealth format without in-person support from clinicians. Inappropriate mealtime behavior includes different topographies (e.g., head turns, batting) that caregivers would need to identify and respond to. During initial sessions, inappropriate mealtime behavior often occurs at high levels and may include other responses within a hierarchy of refusal behaviors (e.g., crying, aggression), which may be overwhelming. In light of similar challenges with other severe problem behaviors, previous researchers have demonstrated that caregivers can conduct functional analyses of other response topographies via telehealth (e.g., Wacker et al., 2013). Machalicek et al. (2016) taught caregivers to implement functional analyses of challenging behaviors via telehealth and demonstrated that functionbased intervention led to improvements in challenging behaviors for three children. Wacker et al. (2013) taught caregivers of 20 children to implement functional analyses of problem behaviors (e.g., aggression, destructive behaviors, self-injury, repetitive behaviors) via telehealth with parent assistants to provide on-site support. Due to the successful extension of functional analysis of severe problem behaviors to a telehealth format, it is worth exploring a similar extension of functional analysis of inappropriate mealtime behavior to telehealth. Therefore, this study aimed to extend the previous literature on functional analysis of inappropriate mealtime behavior and demonstrate that caregivers can conduct functional analysis of inappropriate mealtime behavior via telehealth.

\section{Method}

\section{Participant}

Rosa was a 4-year-old female with Down syndrome, hypothyroidism, hypertension, chronic lung disease, and a G-tube. She met the criteria for avoidant/restrictive food intake disorder, because she received $100 \%$ of her hydration needs via Gtube. Rosa's cardiologist referred her to a feeding program due to G-tube dependence, failure to meet caloric needs orally, and inappropriate behaviors during meals. She had a history of dependence on G-tube feedings; her last solid food G-tube feeding was 4 months before admission. She consumed $76 \%$ of her caloric needs orally through various pureed food blends when fed by a caregiver with continuous access to an iPad. Rosa had not received any prior feeding therapy in a clinic setting. She participated in an interdisciplinary evaluation with a behavioral psychologist, dietitian, speech-language pathologist, and gastroenterologist. The interdisciplinary team determined that she was a safe oral feeder (i.e., no risks of aspiration, no food allergies). At admission, her weight and height for her age were in the $22^{\text {nd }}$ and $46^{\text {th }}$ percentiles, respectively.

Rosa lived with her mother, father, and sister, who were at home due to the COVID-19 pandemic. Before admission, therapists conducted a phone interview with caregivers to discuss the appropriateness of telehealth services and explain (1) the structure of the program, (2) daily roles and responsibilities (e.g., food prep, feeding meals, meal schedule), and (3) necessary materials (e.g., internet connection, laptop computer, blender). Following review of Rosa's history and records, consultation with the interdisciplinary team, and agreement from the caregivers, the therapists determined telehealth services were appropriate.

\section{Setting and Materials}

All sessions were conducted via a HIPPA-compliant video conferencing software (i.e., Zoom). Rosa's mother and father conducted all sessions at their kitchen table. Rosa's sister was not involved in sessions. Materials for therapists included laptop computers, DataPal 1.0 data recording software (i.e., a beta version of BDataPro; Bullock et al., 2017), and timers. Materials for caregivers included a booster chair, small maroon spoons, bowls, pink cut-out cups, syringes, a scale, a bib, and preferred toys. Therapists selected 4 of the 16 caregiverselected foods (pureed chicken breast, green beans, potatoes, and pears) to include in solids sessions. Caregivers selected chocolate Carnation Breakfast Essentials as the liquid to target.

\section{Dependent Variables}

Therapists used DataPal 1.0 to record the frequency of inappropriate mealtime behavior when the food, liquid, utensil, or cup was within Rosa's reach and she (1) turned her head 45 degrees or moved $5 \mathrm{~cm}$ away from the utensil or cup, (2) touched the utensil, cup, or any part of the feeder's arm, or (3) covered her mouth. Therapists also recorded session time as the duration of time the food, liquid, utensil, or cup was within Rosa's reach (i.e., the opportunity to engage in inappropriate mealtime behavior was present). Therapists determined the rate of inappropriate mealtime behavior per min by dividing the frequency of inappropriate mealtime behavior by the session time.

\section{Procedural Integrity}

Therapists collected data on correct procedure at the end of each bite or drink interval for $100 \%$ of solids and liquids sessions. Therapists recorded correct procedure if the caregiver implemented the protocol within $3 \mathrm{~s}$ of the time outlined in the written instructions for each bite or drink interval or if the 
caregiver changed behavior immediately after feedback from the therapist following errors of omission. To be recorded as correct procedure, the caregiver must have (1) presented the bite or drink to the child's lips, (2) kept the utensil or cup where it was presented for the duration of the trial (except during escape conditions), (3) withheld attention and tangibles during all test conditions (unless following inappropriate mealtime behavior during the respective test condition), (4) provided free access to attention and tangibles in control conditions, and (5) delivered the programmed consequence following inappropriate mealtime behavior (i.e., removed utensil or cup, provided attention, or provided a toy). Correct procedure was $100 \%$ for solids and liquids sessions.

\section{Interobserver Agreement}

Two trained therapists simultaneously collected data on inappropriate mealtime behavior and correct procedure for $85 \%$ of solids and $40 \%$ of liquids sessions. Therapists calculated mean count-per-interval interobserver agreement (IOA) for inappropriate mealtime behavior and total count IOA for correct procedure. Mean count-per-interval IOA was calculated by dividing the smaller count by the larger count in each 10-s interval and converting the ratio to a percentage. Therapists averaged the percentage for each interval to determine an agreement score for each session. Total count IOA was calculated by dividing the smaller count by the larger count in each session and converting the ratio to a percentage. IOA for inappropriate mealtime behavior averaged 90\% (range: $49 \%-100 \%$ ) and $74 \%$ (range: $45 \%-100 \%$ ) for solids and liquids, respectively. IOA for correct procedure averaged 99\% (range: 80\%-100\%) and $92 \%$ (range: $60 \%-100 \%$ ) for solids and liquids, respectively.

\section{Experimental Design}

We used a pairwise design (Bachmeyer et al., 2009) to compare inappropriate mealtime behavior per min in control and test conditions. Therapists determined the order of phases and the order of test and control conditions within each phase randomly (random.org/lists/).

\section{Procedures}

Therapists and caregivers conducted five 30- to 40-min meals per day. Rosa's mother and father fed both solids and liquids functional analyses. Each session consisted of five bite or drink presentations. For solids sessions, the caregiver presented a level small maroon spoon bite of each food (approximately $2-3 \mathrm{cc}$ ), with one food presented twice randomly. For liquids sessions, the caregiver presented $2 \mathrm{cc}$ of chocolate Carnation Breakfast Essentials in a pink cut-out cup for all five drinks.
Before the functional analysis, therapists coached the caregivers through conducting a multiple stimulus without replacement preference assessment to determine highly preferred items (DeLeon \& Iwata, 1996). Rosa's highest preferred items were the iPad, a toy doctor's kit, and a telephone.

\section{Feeder Training}

Therapists provided caregivers with an electronic copy of the functional analysis procedures via email. Before implementing each condition, therapists explained the procedures and answered questions. Given the similarities across conditions, therapists explained the procedures for one condition at a time to limit confusion. Throughout the functional analysis, therapists provided brief instructions before each session, real-time remote coaching following errors verbally or via the chat function on Zoom, and praise for correct procedure.

\section{Functional Analysis}

General procedures Functional analysis conditions included control, attention, escape, and tangible. A tangible condition was included because therapists observed caregivers deliver items contingent on inappropriate mealtime behavior during initial meal observations (Rooker et al., 2011). Procedures were similar to those described by Kirkwood et al. (in press). During each bite or drink presentation, the caregiver touched the spoon or cup to the child's lips and prompted the child to "take a bite (drink)." If the child (1) opened her mouth or (2) opened her mouth and moved toward the utensil or cup when crying, the caregiver deposited the food or liquid into her mouth. If the deposit occurred within $5 \mathrm{~s}$ of presentation, the caregiver provided brief praise (e.g., "good job taking your bite"). Therapists started a 30-s timer immediately after deposit. If the bite (drink) was not deposited, it remained where it touched the child's lips for $30 \mathrm{~s}$ before a new bite (drink) was presented. After the timer elapsed, the caregiver checked the child's mouth to determine if she had swallowed the food or liquid. If there was no food or liquid in her mouth, the caregiver provided praise. If there was food or liquid in her mouth, the caregiver reminded her to swallow and presented the next bite or drink. After the fifth bite or drink, if she still had food or liquid in her mouth, the caregiver continued to check her mouth every $30 \mathrm{~s}$ until she swallowed or the session cap (10 min) was reached, at which point the caregiver wiped out her mouth.

Control At the start of each session, the caregiver placed Rosa's preferred items in front of her and instructed her to select one. After selection of an item, the caregiver gave the item to Rosa and provided noncontingent attention (e.g., conversation, singing) throughout the session. The spoon or cup 
remained where it originally touched her lips. After $30 \mathrm{~s}$, the caregiver removed the bite or drink and presented the next bite or drink.

Attention If Rosa engaged in inappropriate mealtime behavior, the caregiver provided attention for $30 \mathrm{~s}$ in the form of coaxing (e.g., "it is just a little bite, you can do it") and comforting statements (e.g., "you are okay"). If Rosa did not engage in inappropriate mealtime behavior, the caregiver remained silent for $30 \mathrm{~s}$. The utensil or cup remained where it originally touched her lips for $30 \mathrm{~s}$, at which point the caregiver removed the bite or drink and presented the next bite or drink.

Escape If Rosa engaged in inappropriate mealtime behavior, the caregiver removed the bite or drink for $30 \mathrm{~s}$. If Rosa did not engage in inappropriate mealtime behavior, the caregiver kept the utensil or cup where it originally touched her lips. After $30 \mathrm{~s}$, the caregiver presented the next bite or drink.

Tangible If Rosa engaged in inappropriate mealtime behavior, the caregiver provided the highest preferred item from the preference assessment (i.e., iPad). The caregiver allowed Rosa to interact with the item for $30 \mathrm{~s}$ and kept the bite or drink where it originally touched her lips. If Rosa did not engage in inappropriate mealtime behavior, the caregiver did not provide the item. After $30 \mathrm{~s}$, the caregiver removed the item and presented the next bite or drink.

\section{Data Analysis}

We used visual inspection to determine if test and control conditions were differentiated. After each pair of test and control conditions, we visually inspected the level, trend, and variability of inappropriate mealtime behavior in the test condition compared to the control condition. Once two therapists reached a consensus on the differentiation between test and control conditions, therapists evaluated the next putative reinforcer or ended the analysis.

\section{Results}

Figure 1 depicts the results of Rosa's functional analyses for solids (top panel) and liquids (bottom panel). For solids, rates of inappropriate mealtime behavior were higher in the escape $(M=17.3$; range: $17.1-17.4)$, tangible $(M=7$; range: $4.7-$ $11.5)$, and attention ( $M=5.6$; range: $3.2-8.4)$ conditions compared to the control condition $(M=1.1$; range: $0-6.5)$. For liquids, rates of inappropriate mealtime behavior were higher in the escape condition ( $M=26.7$; range: $7.0-50)$ compared to attention $(M=6.4$; range: $4.8-8.2)$, tangible $(M=11.7$; range: 7.9-17.1), and control ( $M=11.3$; range: 4.6-19.1) conditions.
These results suggest that Rosa's inappropriate mealtime behavior was maintained by escape, attention, and tangibles during solids meals and escape during liquids meals. The solids and liquids functional analyses were completed in 5 and 10 meals, respectively.

\section{Discussion}

The current study extends previous literature on functional analysis of inappropriate mealtime behavior. Results indicated escape, attention, and tangibles maintained inappropriate mealtime behavior during solids meals, and escape maintained inappropriate mealtime behavior during liquids meals.

At the time of the analyses, Rosa relied on purees for the majority of her caloric needs but did not reliably drink from an open cup; she met her liquid needs via G-tube. It is likely that the caregivers attempted different strategies (e.g., providing tangible items, coaxing) to increase consumption of purees that influenced the maintaining variables of inappropriate mealtime behavior in that context, whereas the introduction of different contingencies had not occurred with liquids. It is possible that Rosa's different histories with solids and liquid led to identifying different functions.

Although the purpose of the current study was not to evaluate caregiver training procedures, an interesting finding is that caregivers did not require all behavioral skills training components to implement the procedures successfully. Similar to findings of previous studies (e.g., Bachmeyer-Lee et al., 2020), caregivers performed each of the protocol steps correctly with fewer components.

Implementation of a functional analysis may be possible via telehealth, but adequate preparation and evaluation of the risks and benefits to all involved are necessary. Clinicians should prepare caregivers in advance by explaining the purpose, procedures, and expectations of the assessment, as well as how the child may respond. In addition, multiple therapists are available in clinical settings to assist and rotate with one another to prevent burnout, which has the potential to affect treatment integrity negatively (Wehby, 2012). Clinicians should monitor the intensity of the child's refusal behaviors and caregiver's integrity to determine the appropriateness and continuation of the assessment in telehealth modality.

Clinicians should collaborate with an interdisciplinary team to ensure client safety and to evaluate the appropriateness of telehealth services. For example, a child with a history of complex medical issues or aspiration risks would require increased collaboration with an interdisciplinary team. Implementation of the procedures should be supervised closely by behavior analysts or licensed psychologists with expertise in conducting functional analyses of inappropriate mealtime behavior. In addition, clinicians should consider 
Fig. 1 Functional Analyses for Solids and Liquids. Note. These graphs display the rate of inappropriate mealtime behavior per min across sessions of the solids and liquids functional analyses. Responding during the control, escape, attention, and tangible conditions are depicted by closed circles, open squares, open triangles, and open diamonds, respectively

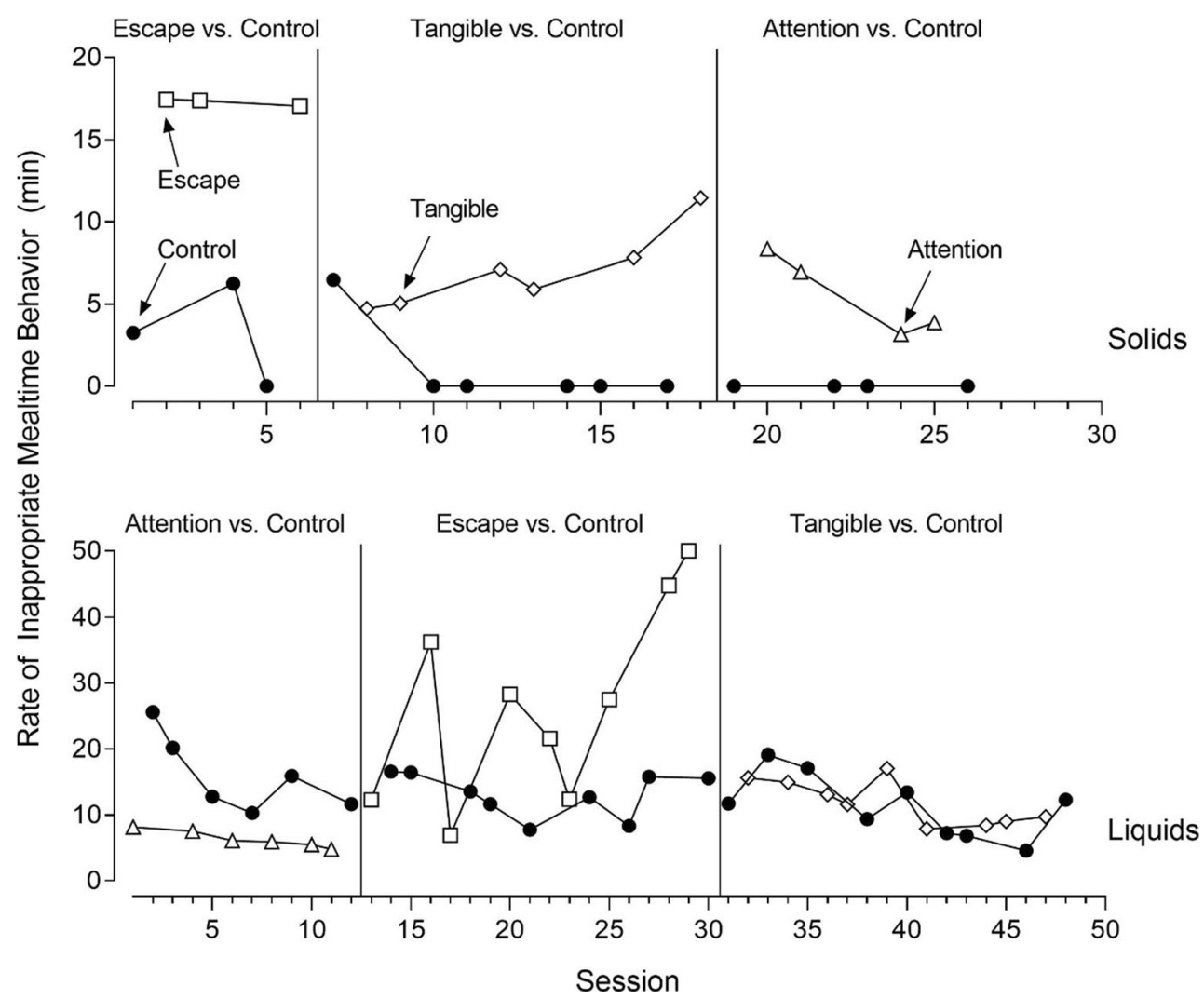

caregiver safety training when considering telehealth services, such as First Aid and cardiopulmonary resuscitation (CPR).

Although this study extends the literature, certain limitations should be noted. First, the caregiver's acceptability of caregiver-implemented functional analyses and of the telehealth format was not directly assessed. It would be important to determine if caregivers prefer clinic-based or therapist-fed assessments in less unique situations when there are more service delivery options available. Second, the definition for correct procedure did not allow us to identify the times in which the caregiver's implementation of a protocol step was delayed or followed a therapist prompt. Last, there were a small number of sessions with low interobserver agreement, which may have been a product of connectivity issues or other common challenges (e.g., audio delays, video freezing, pixilated images) known to occur when delivering services via telehealth (Lee et al., 2014).

Demonstrating that caregivers can implement assessments via telehealth in the home, in the context in which inappropriate mealtime behaviors occur, may serve many benefits. Conducting assessments via telehealth has the potential to increase access to services if future researchers are able to demonstrate that there are feasible treatments to implement in this format. Families would not need to travel to specialized clinics to receive services if clinicians can teach caregivers to implement procedures from their homes. Researchers should extend the current study by evaluating the effectiveness of function-based treatments implemented by caregivers via telehealth. It will be necessary to determine which treatments may be appropriate to train caregivers to implement without the support that is provided in the clinic setting.

In conclusion, results of the current study suggest that caregivers can implement functional analysis procedures to identify maintaining variables of inappropriate mealtime behavior via telehealth. Nevertheless, behavior analysts with experience assessing and treating pediatric feeding problems should determine the appropriateness of providing services via telehealth.

Funding No funding was associated with this study.

\section{Declarations}

Ethical Approval All procedures were performed in accordance with the ethical standards of the institutional review committee.

Conflict of Interest The authors declare no conflict of interest regarding this manuscript.

\section{References}

Bachmeyer, M. H., Kirkwood, C. A., Criscito, A. B., Mauzy IV, C. R., \& Berth, D. P. (2019). A comparison of functional analysis methods of 
inappropriate mealtime behavior. Journal of Applied Behavior Analysis, 52(3), 603-621.

Bachmeyer, M. H., Piazza, C. C., Fredrick, L. D., Reed, G. K., Rivas, K. D., \& Kadey, H. J. (2009). Functional analysis and treatment of multiply controlled inappropriate mealtime behavior. Journal of Applied Behavior Analysis, 42(3), 641-658.

Bachmeyer-Lee, M. H., Kirkwood, C. A., Sheehan, C. M., Gibson, A. L., Shuler, N. J., \& Keane, J. A. (2020). Utilizing in-vivo feedback for caregiver training of pediatric feeding protocols. Journal of Applied Behavior Analysis, 50(3), 1-16.

Bloomfield, B. S., Fischer, A. J., Clark, R. R., \& Dove, M. B. (2019). Treatment of food selectivity in a child with avoidant/restrictive food intake disorder through parent teleconsultation. Behavior Analysis in Practice, 12(1), 33-43.

Bullock, C. E., Fisher, W. W., \& Hagopian, L. P. (2017). Description and validation of a computerized behavioral data program: "BDataPro." The Behavior Analyst, 40(1), 275-285.

Centers for Disease Control \& Prevention (CDC). (2020). How to protect yourself \& others. https://www.cdc.gov/coronavirus/2019-ncov/ prevent-getting-sick/prevention.html

Cox, D. J., Plavnick, J. B., \& Brodhead, M. T. (2020). A proposed process for risk mitigation during the COVID-19 pandemic. Behavior Analysis in Practice, 13(2), 1-7.

DeLeon, I. G., \& Iwata, B. A. (1996). Evaluation of a multiple-stimulus presentation format for assessing reinforcer preferences. Journal of Applied Behavior Analysis, 29(4), 519-533.

Kirkwood, C. A., Piazza, C. C., \& Peterson, K. M. (in press). A comparison of function- and nonfunction-based extinction for inappropriate mealtime behavior. Journal of Applied Behavior Analysis.

Lee, J. F., Schieltz, K. M., Suess, A. N., Wacker, D. P., Romani, P. W., Lindgren, S. D., Kopelman, T. G., \& Dalmau, Y. C. (2014). Guidelines for developing telehealth services and troubleshooting problems with telehealth technology when coaching parents to conduct functional analyses and functional communication training in their homes. Behavior Analysis in Practice, 8(2), 190-200.

Machalicek, W., Lequia, J., Pinkelman, S., Knowles, C., Raulston, T., Davis, T., \& Alresheed, F. (2016). Behavioral telehealth consultation with families of children with autism spectrum disorder. Behavioral Interventions, 31(3), 223-250.

Peterson, K. M., Ibañez, V. F., Volkert, V. M., Zeleny, J. R., Engler, C. W., \& Piazza, C. C. (2021). Using telehealth to provide outpatient follow-up to children with avoidant/restrictive food intake disorder. Journal of Applied Behavior Analysis, 54(1), 6-24.

Rooker, G. W., Iwata, B. A., Harper, J. M., Fahmie, T. A., \& Camp, E. M. (2011). False-positive tangible outcomes of functional analyses. Journal of Applied Behavior Analysis, 44(4), 737-745.

Volkert, V. M., Peterson, K. M., Zeleny, J. R., \& Piazza, C. C. (2014). A clinical protocol to increase chewing and assess mastication in children with feeding disorders. Behavior Modification, 38(5), 705729.

Volkert, V. M., \& Piazza, C. C. (2012). Pediatric feeding disorders. In P. Sturmey \& M. Hersen (Eds.), Handbook of evidence-based practice in clinical psychology, Vol 1: Child and adolescent disorders (pp. 323-337). Wiley https://doi.org/10.1002/9781118156391. ebcp001013.

Wacker, D. P., Lee, J. F., Dalmau, Y. C., Kopelman, T. G., Lindgren, S. D., Kuhle, J., Pelzel, K. E., \& Waldron, D. B. (2013). Conducting functional analyses of problem behavior via telehealth. Journal of Applied Behavior Analysis, 46(1), 31-46.

Wehby, J. H., Maggin, D. M., Partin, T. C. M., \& Robertson, R. (2012). The impact of working alliance, social validity, and teacher burnout on implementation fidelity of the good behavior game. School Mental Health, 4(1), 22-33.

Publisher's Note Springer Nature remains neutral with regard to jurisdictional claims in published maps and institutional affiliations. 